\title{
Modelado borroso mediante datos de entrada-salida obtenidos con Arduino. Aplicación a una pila de combustible
}

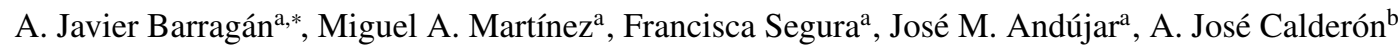 \\ ${ }^{a}$ Escuela Técnica Superior de Ingeniería, Universidad de Huelva. \\ ${ }^{b}$ Escuela de ingenierías Industriales, Universidad de Extremadura.
}

\begin{abstract}
Resumen
Los sistemas hardware de bajo coste se están convirtiendo en una alternativa real y práctica a los sistemas de control comerciales, especialmente por su versatilidad y facilidad de programación, unidas, por supuesto, al bajo precio y alta conectividad de dichos dispositivos. Evidentemente, un hardware de bajo coste tiene unas limitaciones respecto al hardware más comercial y costoso, y estas limitaciones deben conocerse y, sobre todo, tenerse en cuenta por su influencia en el sistema donde se vayan a implantar. En este trabajo se estudia la aplicación de un sistema de bajo coste para la obtención de datos para el modelado de un sistema complejo y no lineal como es una pila de combustible.
\end{abstract}

Palabras Clave:

Arduino, control inteligente, hardware bajo coste, modelado borroso, pila de combustible, sistemas borrosos

\section{Introducción}

Una pila de combustible es un sistema complejo formado por un stack (combinación serie de celdas donde se produce la corriente eléctrica mediante una reacción electroquímica), unos filtros y sistemas acondicionadores de los gases intervinientes en la reacción $\left(\mathrm{H}_{2}\right.$ y $\left.\mathrm{O}_{2}\right)$, un sistema de refrigeración y, por supuesto, un sistema de control. En comparación con otras tecnologías, como la eólica o la generación fotovoltaica, las pilas de combustible puede situarse en cualquier lugar, sin restricciones geográficas o ambientales. Además, son muy respetuosas con del medio ambiente, ya que el único subproducto resultante de la generación eléctrica es agua, lo que permite su uso en una amplia gama de aplicaciones. Por ejemplo, en aplicaciones estacionarias, las células de combustible pueden estar conectados a la red eléctrica (Moreira and da Silva, 2009), instalada como generadores independientes (Kirubakaran et al., 2009), u operar en los vertederos y plantas de tratamiento de aguas residuales (Paska et al., 2009). En las aplicaciones de transporte, hay un gran campo de investigación debido a los vehículos eléctricos (ciclomotores, coches, autobuses o camiones), debida a la creciente escasez de combustibles fósiles (Andújar and Segura,

\footnotetext{
*Autor en correspondencia.

Correos electrónicos: antonio. barragan@diesia.uhu.es (A. Javier Barragán), bohorquez@diesia.uhu.es (Miguel A. Martínez), francisca.segura@diesia.uhu.es (Francisca Segura), andujar@diesia.uhu.es (José M. Andújar), ajcalde@unex.es (A. José Calderón)
}

2009, Bertoluzzo and Buja, 2011). Otra aplicación interesante de las células de combustible son las aplicaciones móviles, donde pilas de combustible pueden cambiar el mundo ubicuo proporcionando baterías de larga duración a teléfonos móviles, ordenadores portátiles, etc. (Ross, 2003).

La estructura básica de una pila de una sola célula, o unidad de PEM, sobre la base de la cual se construye una pila de combustible, consiste en una capa de electrolito en contacto con un ánodo y un cátodo en ambos lados . Una célula de combustible PEM produce energía electroquímica cuando un gas rico en hidrógeno pasa a través del ánodo y un gas rico en oxígeno (o aire) pasa a través del cátodo, con un electrolito entre el ánodo y el cátodo, que permite el intercambio de carga eléctrica (iones). La disociación de las moléculas de hidrógeno produce el flujo de iones a través del electrolito y una corriente eléctrica a través de un circuito externo. El único residuo generado es agua pura. En condiciones de funcionamiento normales, una sola célula produce aproximadamente 1,2 V. Para el uso en sistemas de generación de energía, donde se requiere una potencia relativamente alta, se conectan varias células en serie, formando una pila que puede suministrar energía eléctrica. Las células de combustible PEM están disponibles comercialmente en una amplia gama de potencias (desde pocos $\mathrm{W}$ hasta algunos MW), lo que permite su uso en un gran número de aplicaciones Andújar and Segura (2009).

Una pila de combustible se comporta dinámicamente como un sistema no lineal que genera energía eléctrica no regulada por una reacción electroquímica. Por lo tanto, el uso eficiente 
de la energía requiere una regulación. De esta forma, para la aplicación formal de estrategias de control sobre una célula de combustible, es muy importante contar con un modelo de esta Amphlett et al. (1995, 1996), Famouri and Gemmen (2003), Kim et al. (1995), Van Bussel et al. (1998). En la práctica, el control de la potencia de salida de una pila de combustible se realiza a través de un convertidor $\mathrm{CC} / \mathrm{CC}$ conectado en sus terminales Alam and Tanrioven (2005), Andújar et al. (2011), Segura et al. (2011), Sendjaja and Kariwala (2011), lo que puede suponer una fuente de ruido eléctrico que perturbe el resto de componentes del sistema.

En este trabajo se va a estudiar la obtención de un modelo no lineal para una pila de combustible mediante hardware de bajo coste, más concretamente un sistema Arduino. Este hardware es especialmente interesante por su escaso tamaño, bajo precio y facilidad de programación, lo que permite su utilización en aplicaciones domésticas, donde la reducción de costes sea un factor importante o en prototipado rápido. La placa que se va a emplear tiene una resolución de 10 bits en sus conversores analógico-digitales (A/D), lo que condicionará la precisión de las medidas realizadas. Por la propias limitaciones del hardware a la hora de tomar y almacenar las muestras, el periodo de muestreo en sistemas de estas características suele sufrir variaciones. Estos dos aspectos combinados pueden provocar errores importantes a la hora de obtener un modelo fiable del sistema, por lo que se requiere la adecuación de las estrategias de modelado para minimizar estos efectos en la medida de lo posible.

El modelado de una planta mediante datos entrada-salida permite obtener modelos más realistas de la planta en estudio, por lo tanto, dentro de su ámbito de aplicación, estos modelos son más precisos que los modelos teórico de la misma. El modelado borroso, especialmente modelado basado en sistemas Takagi-Sugeno (TS), permite obtener modelos de alta precisión con un número pequeño de reglas Wang (1997), Andújar and Barragán (2014), por lo que resulta una herramienta ideal para modelar una planta no lineal como es una pila de combustible.

El artículo se estructura en las siguientes secciones: en primer lugar, se ha realizado un introducción del problema a tratar. A continuación, se presenta la formulación que se empleará en el artículo y las técnicas de modelado. Posteriormente se presenta el sistema de laboratorio empleado y se realizarán los modelo mediante las técnicas anteriores, comparando los resultados obtenidos para cada una de ellas. Finalmente se presentan algunas conclusiones.

\section{Formulación del problema de modelado borroso}

Sea un modelo borroso general de $u_{1} . . u_{n}$ entradas e $y_{1} . . y_{m}$ salidas:

$$
\begin{aligned}
& R^{(l, i)}: \text { Si } u_{1}(k) \text { es } A_{1 i}^{l} \text { y } u_{2}(k) \text { es } A_{2 i}^{l} \text { y } \ldots \text { y } u_{n}(k) \text { es } A_{n i}^{l} \\
& \text { Entonces } y_{i}^{l}(k)=a_{0 i}^{l}+\sum_{j=1}^{n} a_{j i}^{l} u_{j}(k),
\end{aligned}
$$

representa el conjunto de reglas del modelo borroso, donde $l=$ 1.. $M_{i}$ es el índice de la regla y $M_{i}$ el número de reglas de la $i$-ésima salida del sistema, $y_{i}$. Si $\tilde{\mathbf{x}}=\left(1, u_{1}, \ldots, u_{n}\right)^{\mathrm{T}}$ Andújar et al. (2009), el equivalente matemático de este modelo es:

$$
y_{i}(k)=h_{i}(\mathbf{u}(k))=\sum_{j=0}^{n} a_{j i}(\mathbf{u}) \tilde{u}_{j}(k),
$$

donde:

$$
a_{j i}(\mathbf{u})=\frac{\sum_{l=1}^{M_{i}} w_{i}^{l}(\mathbf{u}) a_{j i}^{l}}{\sum_{l=1}^{M_{i}} w_{i}^{l}(\mathbf{u})},
$$

$\mathrm{y}$

$$
w_{i}^{l}(\mathbf{u})=\prod_{j=1}^{n} \mu_{j i}^{l}\left(x_{j}(k), \sigma_{j i}^{l}\right),
$$

siendo $\sigma_{j i}^{l}$ los parámetros adaptables de los antecedentes del modelo borroso, es decir, los parámetros que definen las funciones de pertenencia de los conjuntos borrosos $A_{j i}^{l}$.

Una metodología práctica para obtener modelos de sistemas complejos es emplear algún algoritmo de modelado en base a datos de entrada-salida. Estos algoritmos permiten la obtención de modelos no lineales bastante precisos. ANFIS es uno de los algoritmos más conocidos y empleados para la obtención de modelos borrosos en base a una estructura interna en forma de red neuronal (Jang, 1993).

Otro algoritmo presentado recientemente se basa en el filtro de Kalman (Barragán et al., 2014), y tiene la ventaja de incorporar el conocimiento de ruidos y/o incertidumbres del modelo al proceso de modelado; además de poder ser aplicado en línea con el sistema. El algoritmo de modelado mediante el filtro de Kalman es realmente la ejecución secuencial de dos filtros (Barragán et al., 2010, 2012): en primer lugar, se realiza el ajuste de consecuentes, y posteriormente el ajuste de los antecedentes mediante dos filtros independientes.

Antes de ejecutar el algoritmo de modelado, el modelo debe ser inicializado. Si no se posee información previa sobre el sistema que permita realizar una mejor inicialización, todos consecuentes pueden tomar el valor 0 , o se pueden emplear otros procedimientos de inicialización (Benmakrouha, 1997). Igualmente, los antecedentes se pueden inicializar con una distribución uniforme en cada uno de los universos de discurso de las entradas del modelo borroso, o emplear un conjunto de datos previos y realizar un clustering para obtener las reglas iniciales.

El proceso de modelado borroso trata de obtener un modelo en base a reglas de la forma (1) a partir de los datos del sistema. En este caso, las entradas para el modelo serán tanto las variables de estado, $\mathbf{x}(k)$, como las propias entradas de control del sistema, $\mathbf{u}(k)$; y las salidas serán los valores para el siguiente periodo de muestreo de las variables de estado, $\mathbf{x}(k+1)$.

En este trabajo se van a emplear tanto el algoritmo ANFIS como el filtro de Kalman para comprobar cuál se adapta mejor al modelado a partir de datos obtenidos con sistemas de bajo coste. 


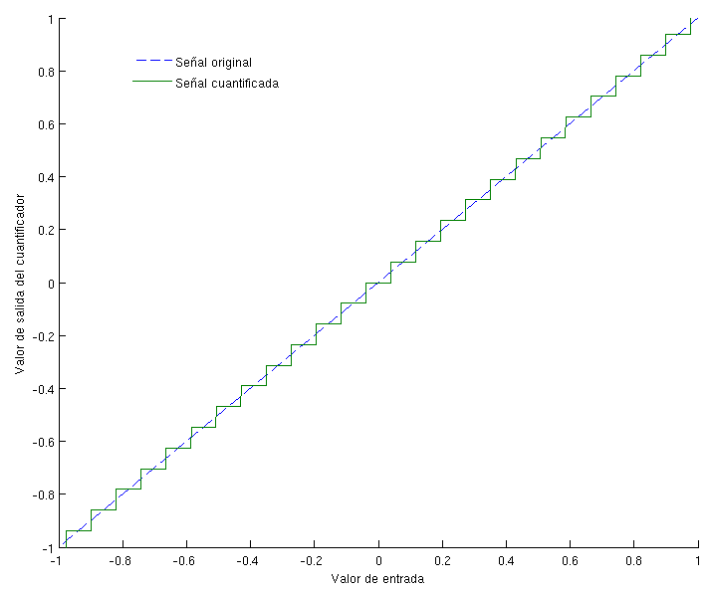

Figura 1: Señal cuantificada vs. señal original para un convertidor A/D de 8 bits y un span de $20 \mathrm{~V}$.

\section{Modelado de la pila de combustible FCgen-1020ACS de Ballard mediante datos obtenidos por hardware de bajo coste}

Para la realización de los modelos se ha empleado una pila FCgen-1020ACS de Ballard. Esta pila es refrigerada por aire, por lo que no se requiere humidificación externa. Por otra parte, la pila está diseñada en configuración dead-ended usando hidrógeno seco, por lo que no se necesita la humidificación de hidrógeno externo, y la presión de hidrógeno de entrada puede variar desde 1,16 bar a 1,56 bar. La pila produce la corriente continua limpia con una baja firma térmica y acústica. El número de células es de 80 , y pueden alcanzar hasta $3,4 \mathrm{~kW}$.

Respecto al sistema de adquisición, se ha empleado un Arduino ATMega256, el cual posee 16 entradas analógicas multiplexadas a un conversor analógico-digital de 10 bits que se conectan a la pila de combustible mediante un circuito de adaptación. El error de cuantificación, $e_{q}$, de un conversor analógicodigital es (Smith, 2007):

$$
e_{q}= \pm \frac{\Delta}{2}
$$

siendo $\Delta$ el escalón de cuantificación, o mínimo valor de la entrada capaz de distinguir el conversor:

$$
\Delta=\frac{\operatorname{span}}{2^{n}}
$$

donde $n$ es el número de bits del conversor A/D. En la figura 1 se muestra a modo de ejemplo el error de cuantificación de una señal de $20 \mathrm{~V}$ de span para un conversor de 8 bits $(\Delta=$ $78,125 \mathrm{mV}$ ).

La varianza del error de cuantificación es (Smith, 2007):

$$
\sigma_{e}^{2}=\frac{\Delta^{2}}{12}
$$

El span del sistema lectura de tensión se ha establecido a través de los circuitos de adaptación en $100 \mathrm{~V}$, por lo que el error de cuantificación para el sistema está entre $\pm 48,828 \mathrm{mV}$, y su varianza es $\sigma_{e}^{2}=0,79473 \mathrm{mV}$. Puesto que el filtro de Kalman puede incorporar la información del ruido del sistema, estos datos pueden emplearse para mejorar el modelado.

Para comprobar el desempeño tanto del algoritmo ANFIS como del filtro de Kalman bajo un muestreo poco regular, y con un error de cuantificación apreciable, se ha realizado el modelado de la pila a partir de un conjunto de datos cuyo periodo de muestreo es de $680 \mathrm{~ms}$, con una desviación típica de $624 \mathrm{~ms}$. La validación se ha realizado con un conjunto distinto con un periodo de muestreo de $724 \mathrm{~ms}$ y una desviación típica de $624 \mathrm{~ms}$. Los resultados de estas pruebas pueden verse en la tabla $1, \mathrm{y}$ gráficamente en las figuras 2 y 3 . Puede comprobarse como el filtro de Kalman obtiene modelos ligeramente mejores que el algoritmo ANFIS. Además, hay que tener en cuenta que este algoritmo es bastante más rápido en su ejecución, y puede ejecutarse en línea con el funcionamiento del proceso.

Tabla 1: Resultados de las pruebas.

\begin{tabular}{lcc}
\hline & ANFIS & Kalman \\
\hline Error absoluto medio de Modelado & 0,02875 & 0,01781 \\
Error absoluto medio de Validación & 0,18663 & 0,11909 \\
\hline
\end{tabular}

\section{Conclusiones}

En este trabajo se han comparado varias métodos de modelado conocidos sobre datos de entrada-salida de una pila de combustible obtenidos por un sistema de adquisición de bajo coste basado en Arduino. Se han empleado dos algoritmos para comprobar su funcionamiento frente a la falta de constancia del periodo de muestreo y frente a errores de cuantificación.

\section{Referencias}

Alam, M. S., Tanrioven, M., May 2005. Modeling and fuzzy logic control of DC-DC converter for proton exchange membrane fuel cell. In: M.H., H. (Ed.), International Conference on Modelling and Simulation, IASTED'05. Cancunm, Mexico, pp. 404-407.

DOI: $10.1016 / \mathrm{j}$.ijhydene.2009.08.090

Amphlett, J. C., Baumert, R. M., Mann, R. F., Peppley, B. A., Roberge, P. R., Harris, T. J., Jan. 1995. Performance modeling of the Ballard Mark IV solid polymer electrolyte fuel cell i. Mechanistic model development. Journal of the Electrochemical Society 142 (1), 1-8. DOI: $10.1149 / 1.2043866$

Amphlett, J. C., Mann, R. F., Peppley, B. A., Roberge, P. R., Rodrigues, A., Feb. 1996. A model predicting transient responses of proton exchange membrane fuel cells. Journal of Power Sources 61 (1-2), 183-188, cited By (since 1996) 216.

DOI: $10.1016 / \mathrm{S} 0378-7753(96) 02360-9$

Andújar, J. M., Barragán, A. J., Apr. 2014. Hibridación de sistemas borrosos para el modelado y control. Revista Iberoamericana de Automática e Informática Industrial \{RIAI\} 11 (2), 127-141. DOI: http://dx.doi.org/10.1016/j.riai.2014.03.004

Andújar, J. M., Barragán, A. J., Gegúndez, M. E., Oct. 2009. A general and formal methodology for designing stable nonlinear fuzzy control systems. IEEE Transactions on Fuzzy Systems 17 (5), 1081-1091. DOI: $10.1109 /$ TFUZZ. 2009.2021984

Andújar, J. M., Segura, F., Dec. 2009. Fuel cells: History and updating. A walk along two centuries. Renewable and Sustainable Energy Reviews 13 (9), 2309-2322.

DOI: $10.1016 / j . r s e r .2009 .03 .015$ 

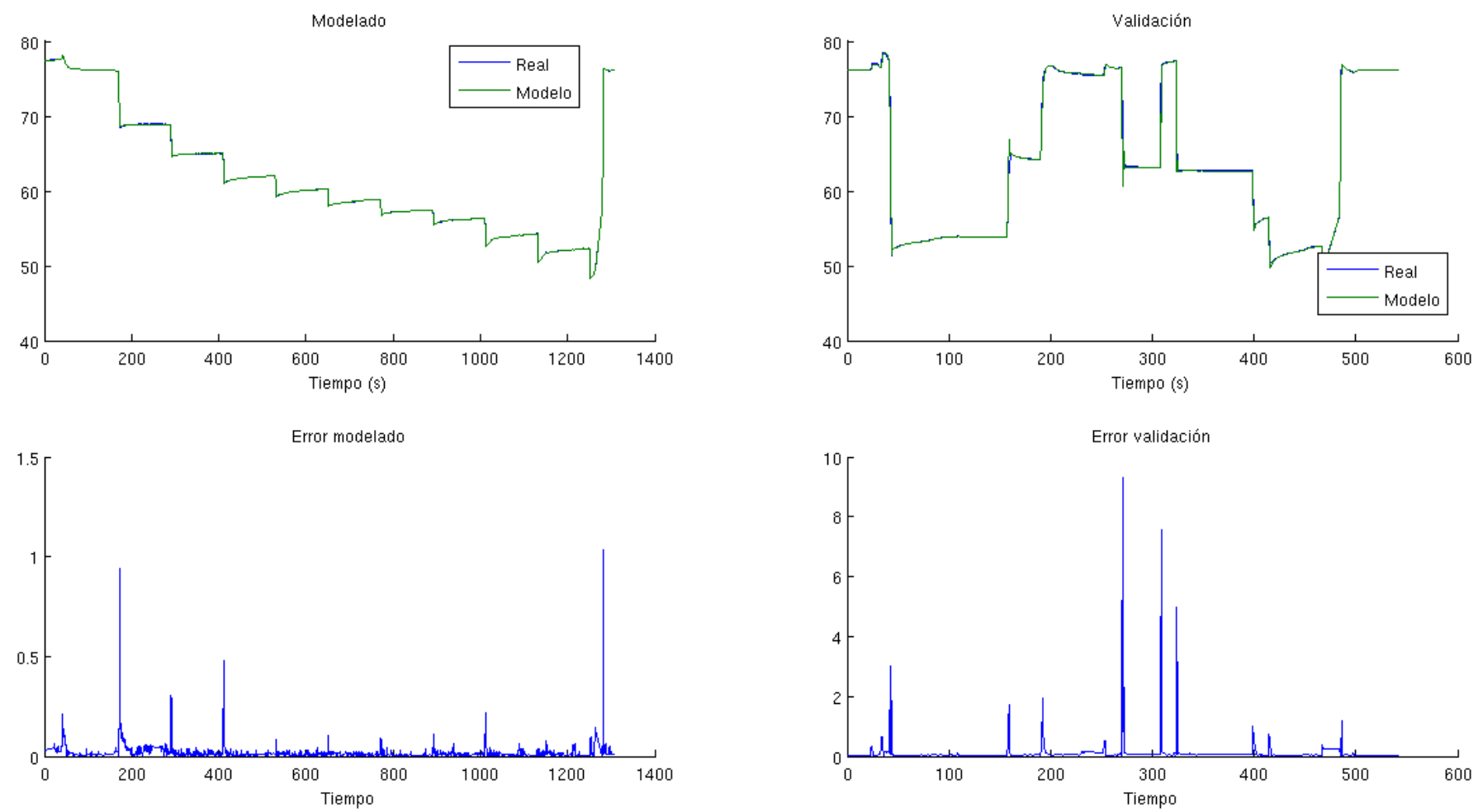

Figura 2: Modelado y validación mediante el filtro de ANFIS.
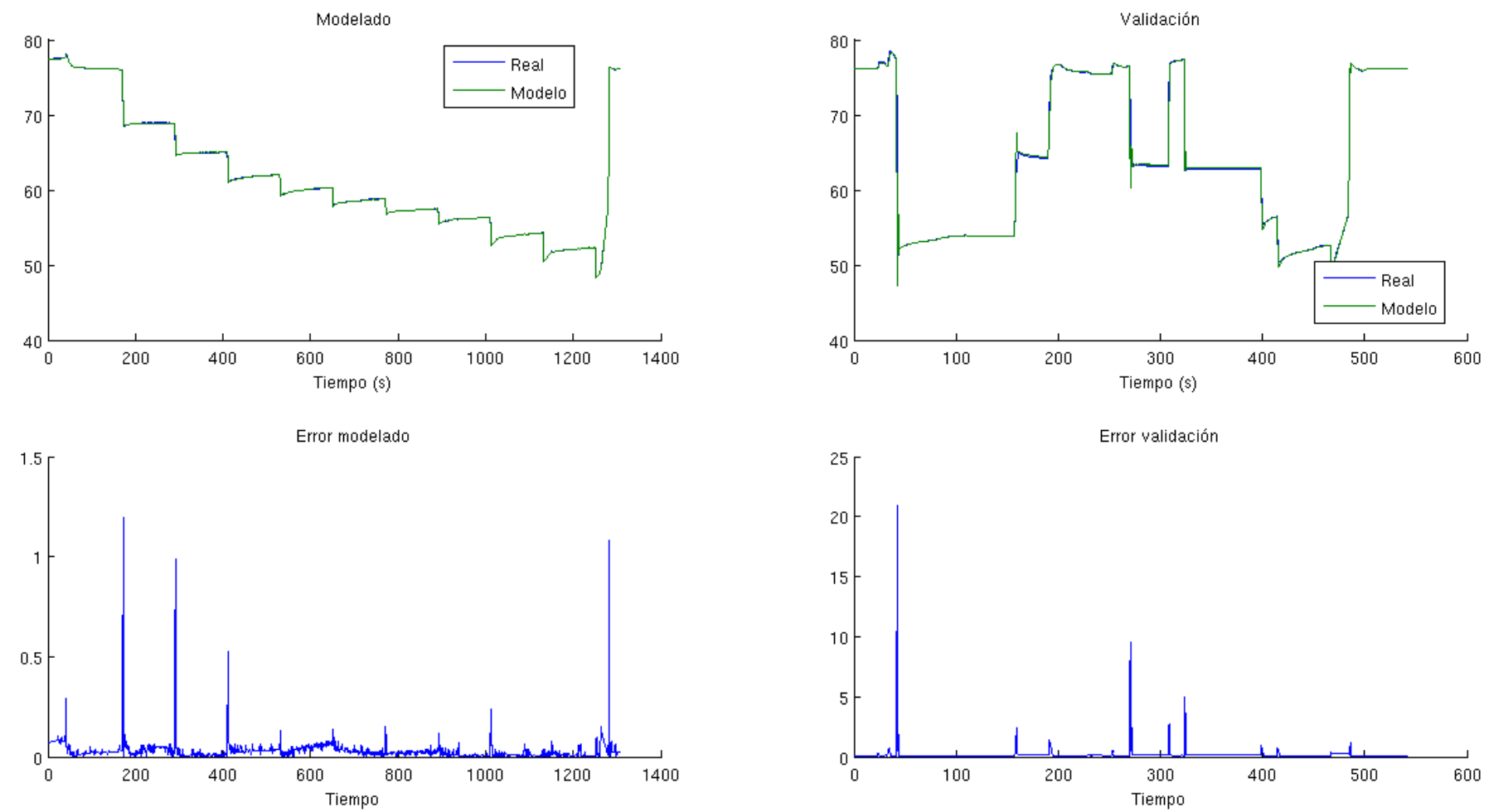

Figura 3: Modelado y validación mediante el filtro de Kalman. 
Andújar, J. M., Segura, F., Durán, E., Rentería, L. A., Nov. 2011. Optimal interface based on power electronics in distributed generation systems for fuel cells. Renewable Energy 36 (11), 2759-2770.

DOI: $10.1016 / j . r e n e n e .2011 .04 .005$

Barragán, A. J., Al-Hadithi, B. M., Jiménez, A., Andújar, J. M., May 2014. A general methodology for online TS fuzzy modeling by the extended kalman filter. Applied Soft Computing 18 (0), 277-289.

DOI: $10.1016 / j$.asoc. 2013.09.005

Barragán, A. J., Andújar, J. M., Jiménez, A., Al-Hadithi, B. M., Sep. 2010. Adaptación paramétrica de un sistema borroso mediante el filtro de Kalman extendido. In: CEA-IFAC (Ed.), XXXI Jornadas de Automática. Jaén, España, pp. 1-6.

Barragán, A. J., Aznar, M., Andújar, J. M., Feb. 2012. Estimador recursivo subóptimo para el ajuste de modelos borrosos ts. In: XVI Congreso Español sobre Tecnologías y Lógica Fuzzy (ESTYLF'12). European Society for Fuzzy Logic and Techonology (EUSFLAT), pp. 354-359.

Benmakrouha, F., 1997. Parameter identification in a fuzzy system with insufficient data. Vol. 1. IEEE, Piscataway, NJ, United States, pp. 537-542. DOI: 10.1109/FUZZY.1997.616424

Bertoluzzo, M., Buja, G., Aug. 2011. Development of electric propulsion systems for light electric vehicles. Industrial Informatics, IEEE Transactions on 7 (3), 428-435.

DOI: $10.1109 /$ TII. 2011.2158840

Famouri, P., Gemmen, R., Jul. 2003. Electrochemical circuit model of a PEM fuel cell. In: Power Engineering Society General Meeting, 2003, IEEE. Vol. 3. pp. 1436-1440. DOI: $10.1109 /$ PES. 2003.1267364

Jang, J.-S. R., May 1993. ANFIS: adaptive-network-based fuzzy inference system. IEEE Transactions on Systems, Man, and Cybernetics 23 (3), 665-685. DOI: $10.1109 / 21.256541$

Kim, J., Lee, S.-M., Srinivasan, S., Chamberlin, C. E., Aug. 1995. Modeling of proton exchange membrane fuel cell performance with an empirical equation. Journal of the Electrochemical Society 142 (8), 2670-2674.
DOI: $10.1149 / 1.2050072$

Kirubakaran, A., Jain, S., Nema, R., Dec. 2009. A review on fuel cell technologies and power electronic interface. Renewable and Sustainable Energy Reviews 13 (9), 2430-2440.

DOI: $10.1016 /$ j.rser. 2009.04.004

Moreira, M. V., da Silva, G. E., Jul. 2009. A practical model for evaluating the performance of proton exchange membrane fuel cells. Renewable Energy 34 (7), 1734-1741.

DOI: $10.1016 / j . r e n e n e .2009 .01 .002$

Paska, J., Biczel, P., Kłos, M., Nov. 2009. Hybrid power systems - an effective way of utilising primary energy sources. Renewable Energy 34 (11), 24142421.

DOI: $10.1016 / j . r e n e n e .2009 .02 .018$

Ross, D., Jul. 2003. Power struggle [power supplies for portable equipment]. IEE Review 49 (7), 34-38. DOI: $10.1049 / \mathrm{ir}: 20030705$

Segura, F., Andújar, J. M., Durán, E., april 2011. Analog current control techniques for power control in PEM fuel-cell hybrid systems: A critical review and a practical application. IEEE Transactions on Industrial Electronics 58 (4), 1171-1184.

DOI: $10.1109 /$ TIE. 2010.2049710

Sendjaja, A., Kariwala, V., May 2011. Decentralized control of solid oxide fuel cells. Industrial Informatics, IEEE Transactions on 7 (2), 163-170. DOI: 10.1109/TII. 2010.2097601

Smith, J. O., 2007. Mathematics of the Discrete Fourier Transform (DFT). W3K Publishing.

Van Bussel, H., Koene, F., Mallant, R. K., Mar. 1998. Dynamic model of solid polymer fuel cell water management. Journal of Power Sources 71 (1-2), 218-222.

DOI: $10.1016 / \mathrm{S} 0378-7753(97) 02744-4$

Wang, L.-X., 1997. A course in fuzzy systems and control. Prentice Hall, New Yersey, USA. 